\title{
A U-shaped actuation system for dual-stage actuators used in hard disk drives
}

\author{
Kenji Suzuki • Eiji Soga • \\ Shinsuke Nakagawa $\cdot$ Toshihiko Shimizu
}

Received: 28 September 2011 / Accepted: 8 June 2012/Published online: 14 August 2012

(c) The Author(s) 2012. This article is published with open access at Springerlink.com

\begin{abstract}
In this paper, we propose an actuation system, called U-shaped actuation, for dual-stage actuator to suppress the arm mode flutter. We manufactured an actuator using this actuation method and measured the frequency response. We found that the frequency response had an inphase arm mode frequency and that there was little difference between each head at arm sway and suspension sway mode. Next, we designed a controller and compared its sensitivity function with that of conventional actuation. By applying stabilizing control to the in-phase arm mode, NRRO of the arm flutter of the outer and inner arms was suppressed by 10.9 and $13.2 \%$, respectively.
\end{abstract}

$\begin{array}{ll}\text { Abbreviations } \\ \text { PZT } & \text { Piezoelectric transducer } \\ \text { VCM } & \text { Voice coil motor } \\ \text { DSA } & \text { Dual stage actuator } \\ \text { NRRO } & \text { Non-repeatable run-out } \\ \text { LDV } & \text { Laser Doppler vibrometer }\end{array}$

K. Suzuki $(\bowtie)$

Hitachi Research Laboratory, Hitachi, Ltd.,

1, Kirihara, Fujisawa, Kanagawa 252-8588, Japan

e-mail: kenji.suzuki.pc@hitachi.com

E. Soga $\cdot$ S. Nakagawa

Hitachi Global Storage Technologies Japan, Ltd.,

1, Kiraiara, Fujisawa, Kanagawa 252-8588, Japan

T. Shimizu

Hitachi Research Laboratory, Hitachi, Ltd.,

832-2, Horiguchi, Hitachinaka, Ibaragi 312-0034, Japan

\section{Introduction}

Hard disk drives are widely used as external storage devices and must provide large capacity and high performance. Use of a wider servo bandwidth for high areal density is limited by the controllability of the voice coil motor (VCM) actuator, so the dual-stage actuator (DSA) is currently being used in hard disk drives. A DSA uses one PZT at each suspension (Fig. 1) and the PZT actuation system affects the frequency characteristic, which is not the case with a VCM actuator. PZT actuation mainly excites two modes: the arm sway mode and the suspension sway mode. Conventional PZT actuation is a reversed-phase system that cancels out the PZT reaction force to avoid exciting the arm mode. However, the frequency responses of different arms behave differently in the suspension sway mode which leads to phase loss at the notch filter.

In addition, arm flutter disturbance over the first resonant mode is an obstacle to accurate DSA head positioning because the disturbance cannot be suppressed by the current PZT actuation system, shown in Fig. 2 (Karaman 2004). From the viewpoint of servo stability, phase-stabilized controller for DSA was studied to suppress the disturbance (Kobayashi 2003; Yunfeng 2002). However, no report has been published on a mechanical PZT actuation method to solve the problem.

In this paper, we propose a better PZT actuation for the dual-stage actuator of hard disk drives and describe a controller design to suppress arm flutter. The PZT actuation direction on the inner arm is the opposite of on the outer arms. We have manufactured an actuator with the proposed PZT actuation and confirmed the advantage of this form of actuation by comparing it with the conventional actuation system. 


\section{PZT actuation system}

\subsection{Conventional actuation system}

We studied the use of a DSA as an actuator for three disk platters. An actuator for three disk platters has two inner arms, two outer arms. The actuator has 6 PZTs and each PZT can actuate in two directions. Therefore, it has 32 combinations of PZT actuation (Fig. 3). In Fig. 3, heads of the outer arms are numbered Head 0 and Head 5, while heads of the inner arms are numbered from Head 1 to Head 4.

At first, we simulated by means of a FEM model to investigate the characteristics of inner and outer arms. In a conventional actuation system (Fig. 4a), the two PZTs of the inner arms actuate in the opposite direction of each other to cancel out PZT reaction forces. Figure 5 shows transfer function from PZT force to head displacement in case of only arm. Thus, the suspension mode of the inner arms is excited, while the arm mode of the outer arm cannot be canceled out by one PZT, as shown in Fig. 5.

Next, we simulated frequency responses of PZT actuation for the three-platter actuator. In Fig. 6, several small

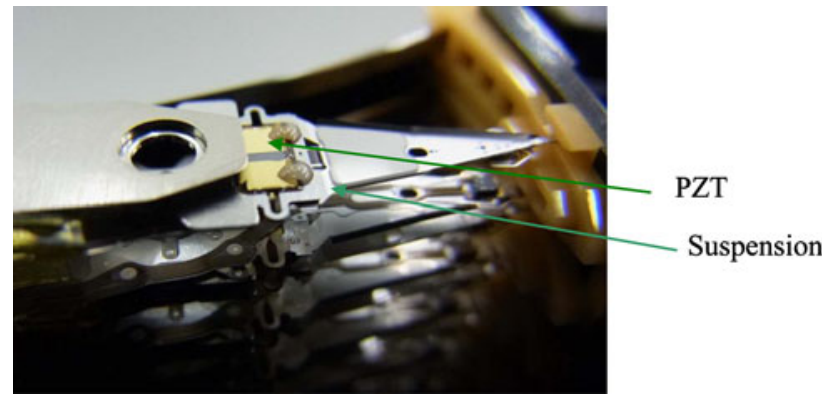

Fig. 1 PZT of dual stage actuator

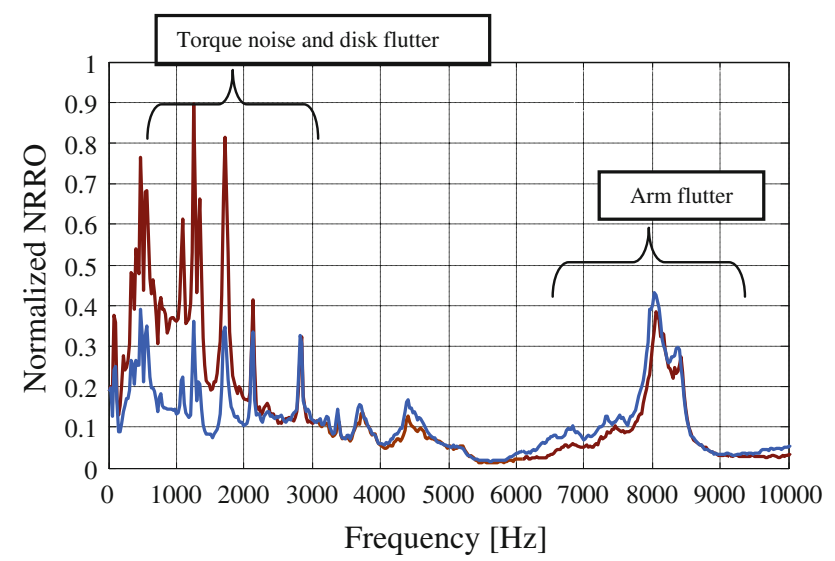

- VCM actuation - DSA actuation

Fig. 2 Comparison of NRRO spectra between VCM actuation and DSA actuation

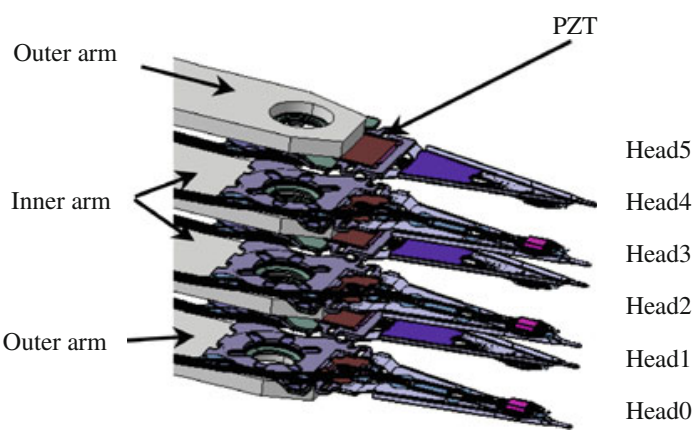

Fig. 3 Definition of head number for all heads

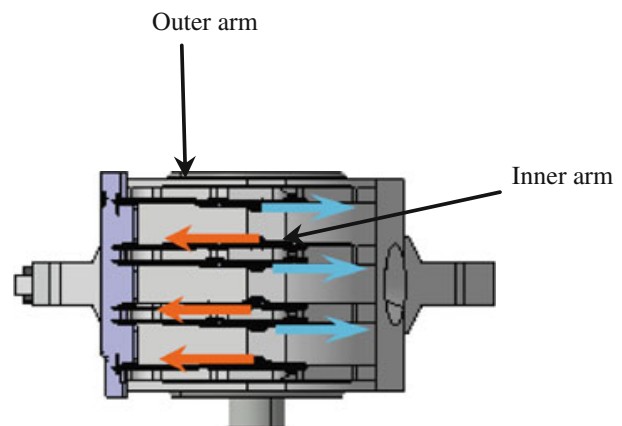

(a) Conventional system

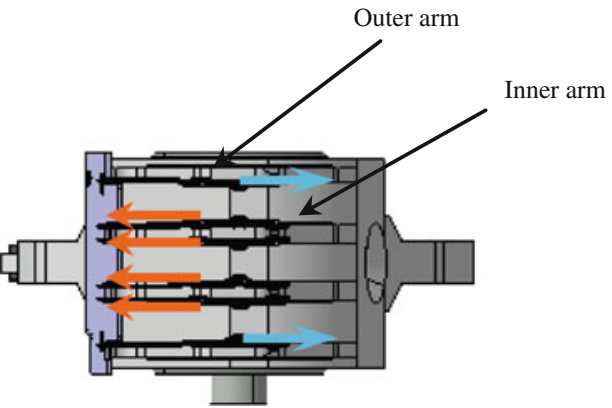

(b) U-shaped system

Fig. 4 Comparison of PZT actuation directions

peaks remain around $8 \mathrm{kHz}$ at heads of all arms because inner and outer arms are coupled with each other unlike in the case of only one arm. In contrast, frequency responses of a conventional actuation system behave differently due to different arms at higher frequency and phase loss for the notch filter being large enough to cover arm mode gain and suspension mode for all heads.

Finally, we measured non-repeatable run-out (NRRO) of the conventional actuation system. NRRO for three disk platters has three peaks around arm modes $(8 \mathrm{kHz})$, shown in Fig. 7. We identified all three arm modes by means of a FEM model and modal shapes of arm modes shown in Fig. 8. First and second components of arm mode NRRO are due to outer arm mode and inner arm mode, 


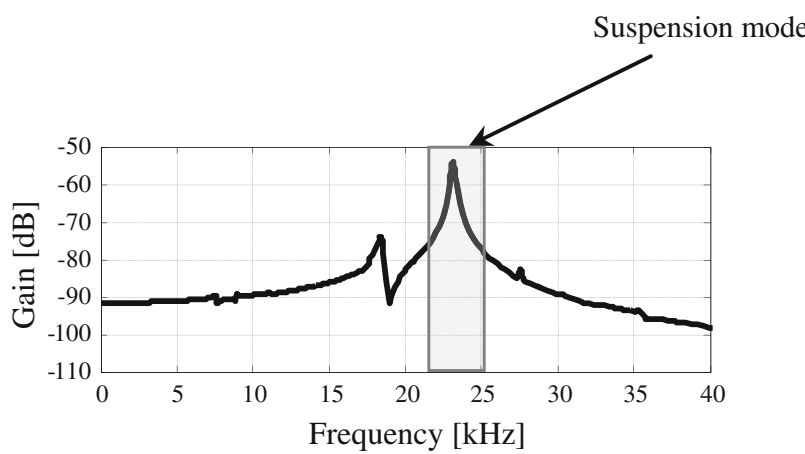

(a) Inner arm

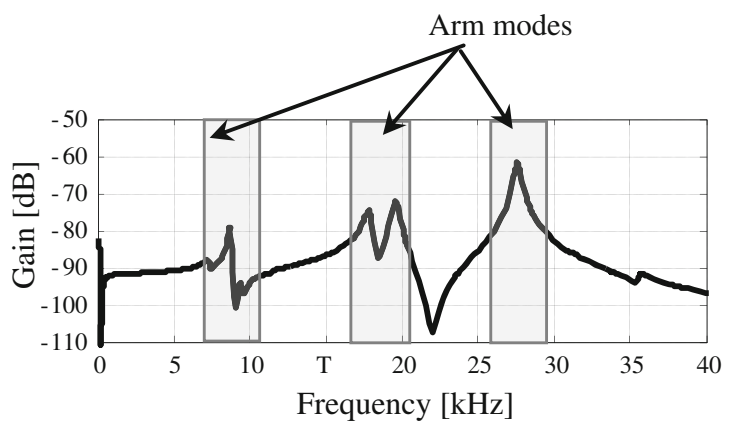

(b) Outer arm

Fig. 5 Frequency response of PZT actuation in case of only arm
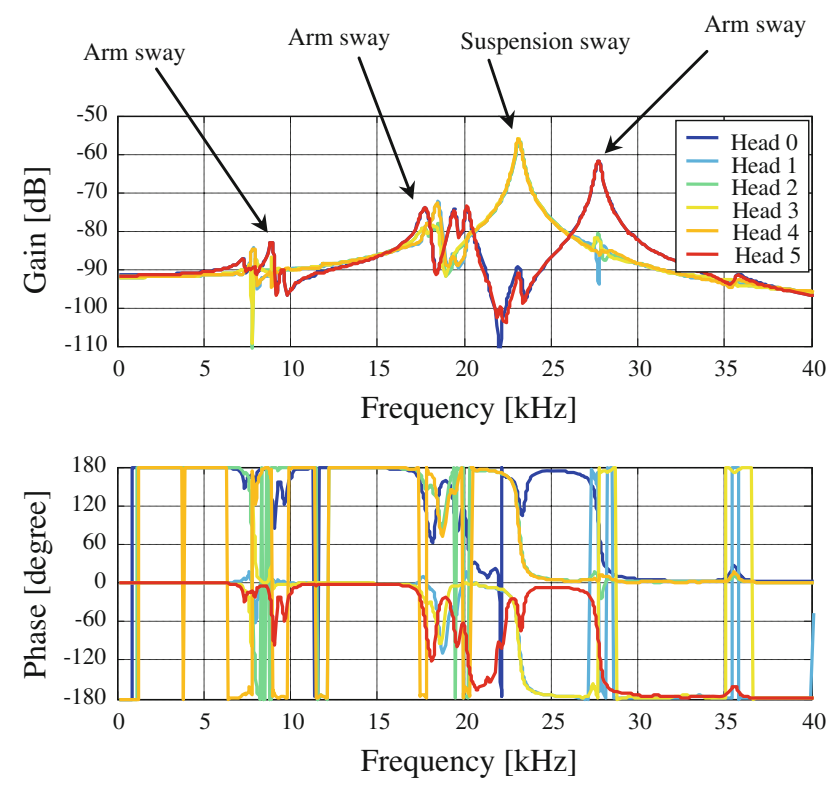

Fig. 6 Frequency response of PZT actuation for three-platter actuator

respectively. The other component is due to in-phase arm mode. Figure 7 shows that the outer and inner arm modes excite NRRO of only outer arm and inner arms, respectively. However, in-phase arm mode excites NRRO of all heads.

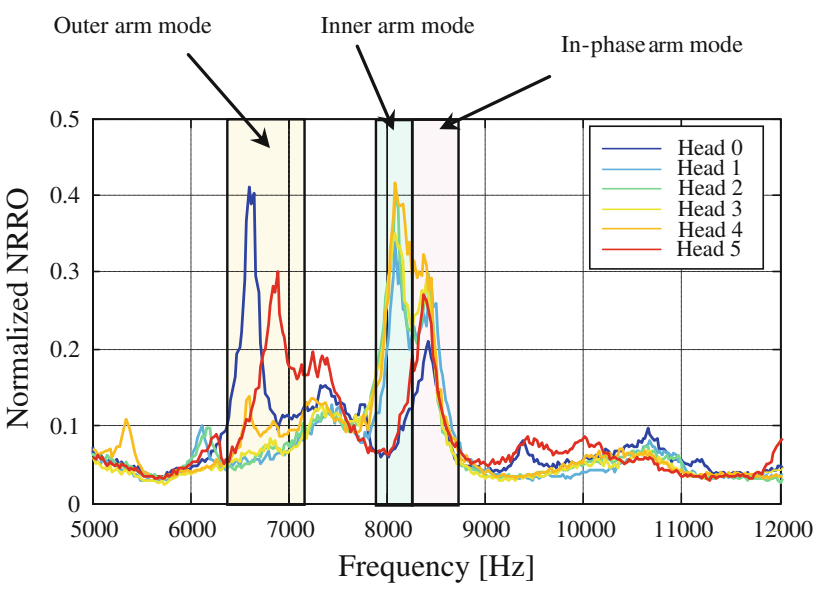

Fig. 7 NRRO spectra of conventional actuation system around arm modes

\subsection{Proposed actuation system}

We propose an actuation system, which we call U-shaped actuation to solve the problem of the conventional actuation system. The PZT actuation directions are compared in Fig. 4. Directions of two PZTs of inner arm are the same, and the proposed actuation excites only arm mode, not suspension mode. In addition, proposed actuation excites in-phase arm mode, because the modal displacement of inphase arm mode is the same as the actuated direction.

We manufactured actuators with conventional and U-shaped actuation and measured the frequency response from PZT voltage to head displacement with a laser Doppler vibrometer (LDV). The frequency responses for conventional and U-shaped actuation are plotted in Figs. 9 and 10, respectively. With the conventional actuation, a few arm modes were exited and the phase of the head on the inner and outer arms differed by about $8 \mathrm{kHz}$ in the arm mode frequency.

Moreover, the frequency responses of the different heads had a suspension sway mode frequency of $23 \mathrm{kHz}$, which led to phase loss caused by the notch filter. In contrast, the U-shaped actuation had an in-phase arm mode frequency, and there was little difference between each head at about $23 \mathrm{kHz}$. Modal shape of the in-phase arm mode is shown in Fig. 8.

\subsection{Controller design}

We designed the controller for two actuation systems. In the U-shaped actuation case, the phase of the main arm mode was kept the same for each arm, so that we could apply stabilizing control for the in-phase arm mode to reduce arm flutter NRRO. Moreover, a notch filter was applied to remove the feedback effect of the suspension mode at $23 \mathrm{kHz}$. Figure 11 plots the average sensitivity functions of all heads. We found that sensitivity at the arm 
Fig. 8 Modal shape and arm displacement of arm modes

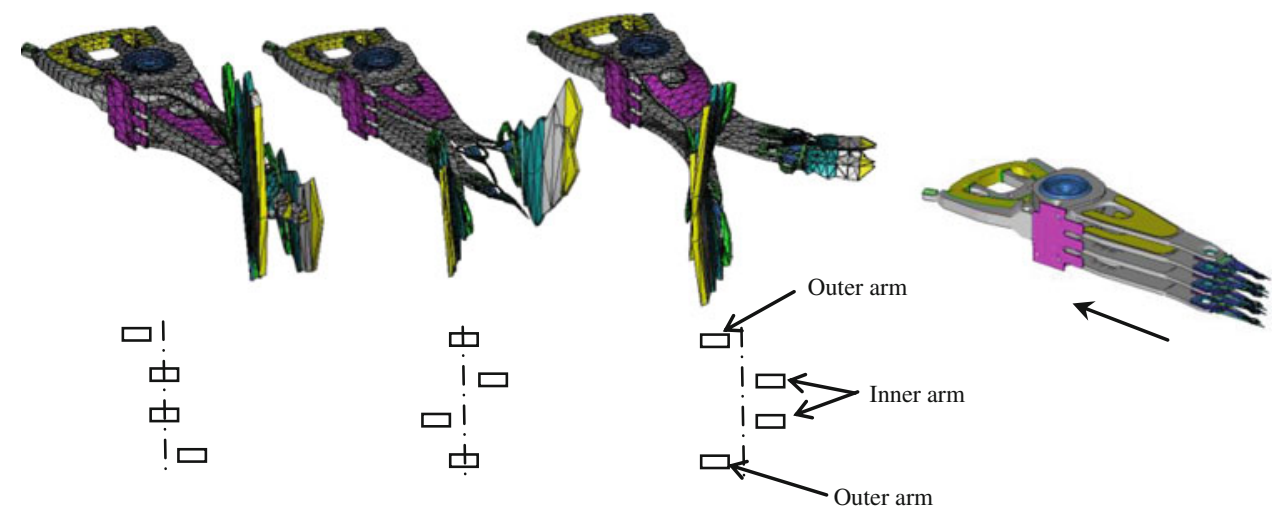
(a) Outer arm mode
(b) Inner arm mode
(c) In-phase arm mode
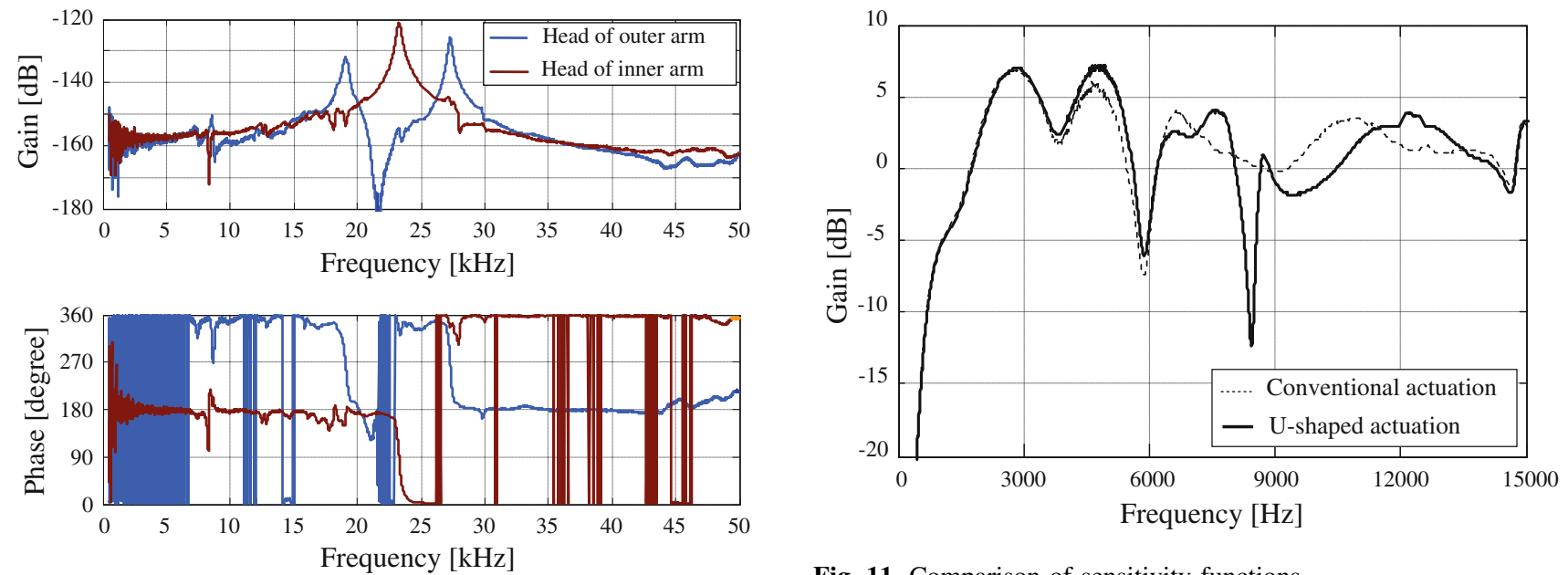

Fig. 9 Frequency response of PZT actuation by conventional system

Fig. 11 Comparison of sensitivity functions
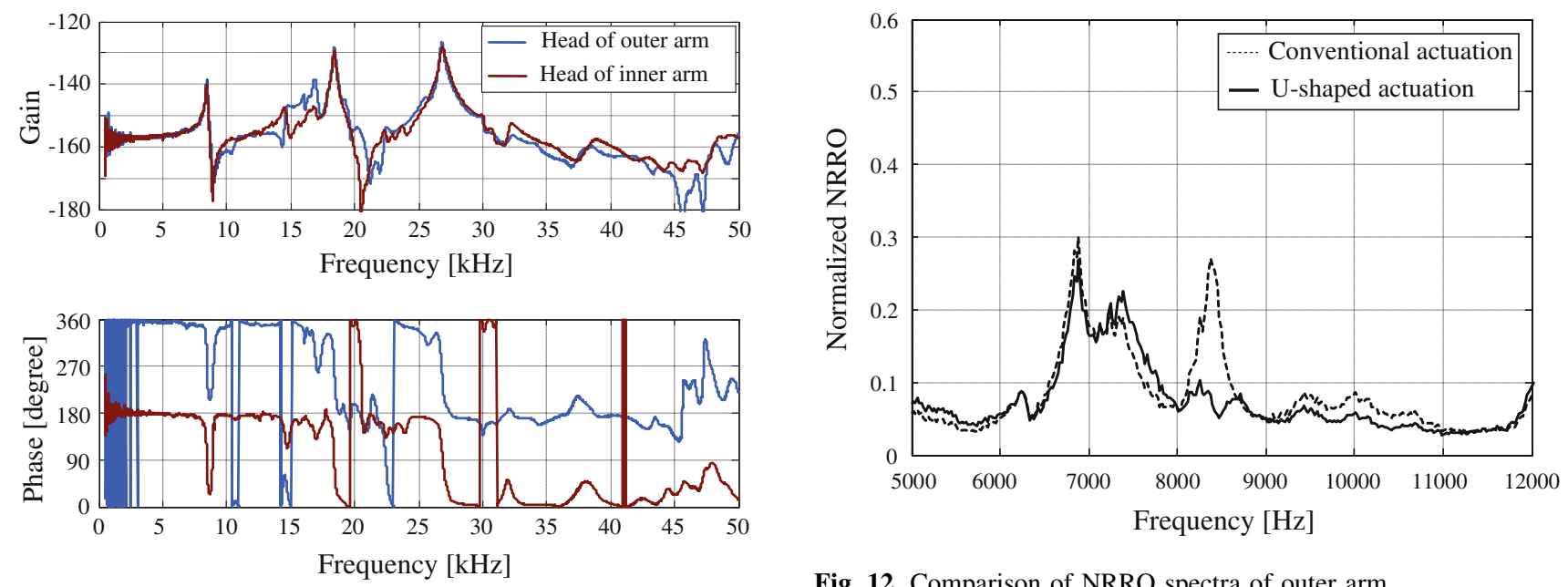

Fig. 12 Comparison of NRRO spectra of outer arm

Fig. 10 Frequency response of PZT actuation by proposed system

mode frequency was suppressed by more than $10 \mathrm{~dB}$ around $8 \mathrm{kHz}$, while the conventional actuation could not suppress the disturbance around arm modes.

Next, we measured the spectrum of non-repeatable runout (NRRO) for conventional actuation. Figures 12 and 13 show NRRO spectra for outer arm and inner arm, respectively. NRRO spectra have some peaks around $8 \mathrm{kHz}$ and 


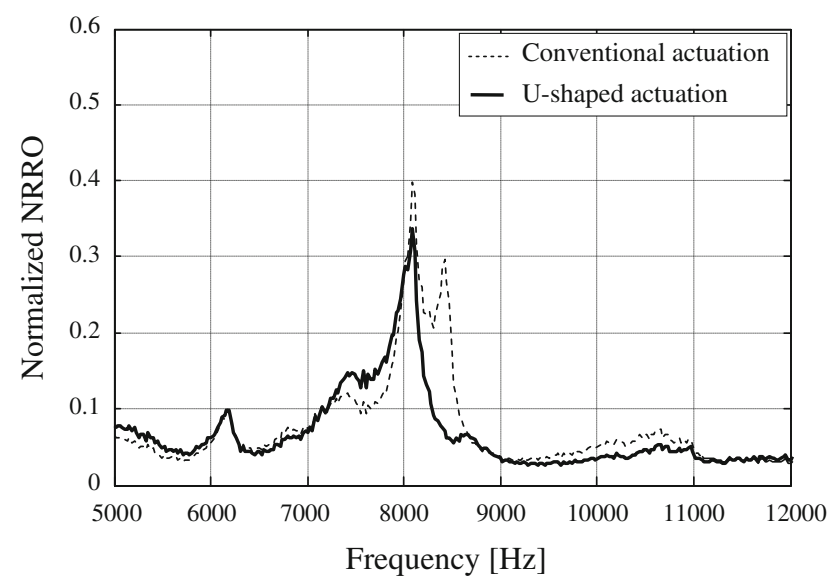

Fig. 13 Comparison of NRRO spectra of inner arm

one of these modes is the in-phase arm mode. We calculated the NRRO spectrum for U-shaped actuation from the difference in the two sensitivity functions. The NRRO spectra are compared in Figs. 12 and 13, which show that the NRRO of the flow-induced arm mode can be suppressed by $10.9 \%$ for the outer arm and $13.2 \%$ for the inner arm compared with those for conventional actuation.

\section{Conclusion}

We have proposed an actuation system, called U-shaped actuation, for dual-stage actuators. We have manufactured an actuator for three platters using this actuation method and measured the frequency response. We found that the frequency response had in-phase arm mode frequency and that there was little difference between each head at arm sway and suspension sway mode. Next, we designed a controller and compared its sensitivity function with that of the conventional actuation. By applying of stabilizing control to the in-phase arm mode, disturbance of in-phase arm mode can be suppressed by around $10 \mathrm{~dB}$. NRRO of the arm flutter of outer and inner arms was suppressed by 10.9 and $13.2 \%$, respectively.

Open Access This article is distributed under the terms of the Creative Commons Attribution License which permits any use, distribution, and reproduction in any medium, provided the original author(s) and the source are credited.

\section{References}

Karaman M (2004) Comparison of suspension-based and slider based microactuators for track following performance. AMC 2004: 347-351

Kobayashi M (2003) A phase-stabilized servo controller for dualstage actuators in hard disk drives. IEEE Trans Magn 39(2):BA4-01-BA4-02

Yunfeng Li (2002) Vibration control of a PZT actuated suspension dual-stage servo system using a PZT sensor, Magnetic Recording Conference, 2002. Digest of the Asia-Pacific, CP6-01 